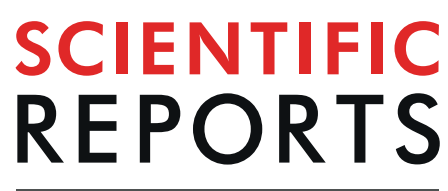

natureresearch

\title{
Low prevalence of symptomatic thyroid diseases and thyroid cancers in HIV-infected patients
}

\author{
Martina Properzi ${ }^{1 *}$, Tommaso della Giustina ${ }^{1}$, Sara Mentasti ${ }^{2}$, Francesco Castelli ${ }^{1}$, \\ Annacarla Chiesa ${ }^{1}$, Natalia Gregori ${ }^{1}$ \& Eugenia Quiros-Roldan ${ }^{1}$
}

Thyroid diseases (TDs) have been widely associated with HIV infection. However, data about TDs prevalence and distribution are controversial, and few published studies are available. The aim of our study was to assess prevalence and risk factors of symptomatic thyroid disturbances, including thyroid cancers, in a large cohort of HIV-infected patients. A retrospective cohort study was performed at the Department of Infectious and Tropical Diseases of the University of Brescia, Italy, in the period 2005-2017. We identified all HIV-positive patients with a diagnosis of symptomatic TD in the electronic database of our Department (HIVeDB); we also operated a record-linkage between our data and the Health Protection Agency database (HPADB) of Brescia Province. Multivariate logistic regression analysis was used to determine risk factors associated with TDs onset; an incidence rate analysis was also performed. During the study period, $6343 \mathrm{HIV}$-infected patients have been followed at our Department; 123 received a diagnosis of symptomatic TD ( $1.94 \%$ of the entire cohort). In the TDs group, almost half of patients were females $(n=59,48 \%)$, mean age was 47.15 years (SD: 11.56$)$. At TD diagnosis, mean T CD4+ cell count was 491 cell/uL and most patients showed undetectable HIV-RNA ( $n=117,95.12 \%)$. Among them, 81 patients were found to have hypothyroidism (63 with Hashimoto's thyroiditis), 21 hyperthyroidism (17 suffered from Graves' disease), while 11 subjects were diagnosed with a primitive thyroid cancer. Papillary thyroid cancer was the most frequent histotype $(n=7,63.63 \%)$, followed by medullary $(n=2,18.18 \%)$ and follicular thyroid cancer $(n=1,9.1 \%)$. Male gender was a protective factor forTDs development, especially for hypothyroidism $(p<0.001)$; age emerged as a variable associated with both hypothyroidism $(p=0.03)$ and thyroid cancer $(p=0.03)$, while CD4+ cell nadir $<200$ cell/ $\mathrm{mm}^{3}$ was associated with symptomatic hyperthyroidism $(p=0.005)$. To conclude, symptomatic thyroid dysfunctions rate in well-treated HIV-infected patients is low. Age and gender are crucial elements in the onset of thyroid abnormalities, together with TCD4+ cell nadir. Interestingly, medullary thyroid cancer seems to be much more frequent in HIV-infected patients compared to the general population.

Various alterations in endocrine homeostasis were described in HIV-infected patients ${ }^{1-3}$. In particular, thyroid diseases (TDs) have been widely associated with HIV infection since the start of the epidemic, long before the beginning of the combination antiretroviral therapy (cART) era ${ }^{4-6}$.

However, there are controversial data concerning thyroid dysfunctions in people living with HIV (PLWH), as previous studies reported both a higher than expected prevalence of $\mathrm{TDs}^{7-13}$ and no apparent increased prevalence of TDs in this population ${ }^{14-17}$. In well-treated HIV-positive individuals, subclinical hypothyroidism ${ }^{7,9,11-13,18}$ and isolated low free thyroxine (FT4) levels ${ }^{6,10,19}$ are the most common described alterations. Anyway, although it is estimated that around one third of PLWH may present biochemical alterations of thyroid function, only 1-3\% develops an overt illness?.

Many factors could be implicated in the pathogenesis of thyroid disturbances in PLWH. Firstly, HIV itself seems to play a crucial role, determining different thyroid alterations over the course of the disease $\mathrm{e}^{5,6}$. Furthermore, several opportunistic infections as from Cytomegalovirus (CMV), Mycobacterium avium, Cryptococcus neoformans, Pneumocystis jirovecii (previously known as P. carinii), Aspergillus spp, Mycobacterium tuberculosis as well as AIDS-defining neoplasms (Kaposi’s Sarcoma, lymphomas) can occasionally involve thyroid

${ }^{1}$ Department of Infectious and Tropical Diseases, University of Brescia and ASST Spedali Civili Hospital, Brescia, Italy. ${ }^{2}$ Department of Medical and Surgical Specialties, Radiological Sciences and Public Health, Unit of Hygiene, Epidemiology and Public Health, University of Brescia, Brescia, Italy. *email: marti.properzi@gmail.com 
gland, rarely resulting in thyroid dysfunction ${ }^{6,20}$. Antiretroviral therapy can also be implicated in TDs. Stavudine has been linked to lower FT4 levels and hypothyroidism ${ }^{7,8,11,19}$, whereas the role of other regimens, as integrase (INIs) and protease inhibitors (PIs), in impairing thyroid function has yet to be fully investigated. Other drugs, as phenytoin, carbamazepine and lithium, that are frequently used among PLWH for psychiatric disorders, can also modify thyroid hormone levels.

Moreover, the improvement of host immune response induced by cART could trigger autoimmune disorders, leading to the appearance of aberrant thyroid autoantibodies. Thus, late after the introduction of antiretroviral therapy, approximately $2 \%$ of PLWH develops Graves' disease ${ }^{14,21,22}$.

Lastly, Hepatitis $\mathrm{C}$ virus (HCV) coinfection is frequently observed in PLWH, due to similar transmission routes. HCV has been associated with different autoimmune conditions, including thyroid disorders, although the actual causative mechanisms have not been entirely determined ${ }^{23,24}$. Furthermore, interferon therapy can also promote autoimmune reactions in $\mathrm{HCV}$-infected patients, especially targeting thyroid gland ${ }^{25}$.

To date, few published papers regarding thyroid dysfunctions in HIV-positive patients are available and thyroid alterations prevalence and distribution in PLWH still remain controversial, since previous studies show sample, definitions and outcomes heterogeneity. Moreover, to our knowledge, no studies focusing only on symptomatic thyroid dysfunctions have been performed and data about thyroid primitive tumors in HIV-infected patients are completely lacking.

Thus, the aims of this study were to assess the prevalence of symptomatic thyroid dysfunctions, including primitive thyroid cancers, in a large cohort of HIV-infected patients in the modern CART era and to evaluate their laboratory and clinical characteristics. Finally, we also determined the risk factors associated with the occurrence of symptomatic thyroid abnormalities in this cohort.

\section{Methods}

Setting and study population. We performed a retrospective cohort study including all HIV-infected patients aged more than 18 years old and receiving cART in charge at the Department of Infectious and Tropical Diseases, ASST Spedali Civili and University of Brescia, Italy. The period of follow-up ranged from January 2005 to December 2017.

We considered as symptomatic TDs all thyroid disorders needing medical or surgical treatment and all thyroid primitive tumors. Symptomatic thyroid dysfunctions were subsequently classified in three groups as follows: hypothyroidisms, hyperthyroidisms and thyroid cancers (TCs).

We identified all HIV-positive patients with a symptomatic TD in the electronic database used for clinical management at our Department (HIVeDB); we also performed a record-linkage between HIV-infected patients followed by our Department and the Health Protection Agency database (HPADB) of Brescia Province, which tracks all services provided by the National Health Service. In this way, we captured all subjects with HIV infection who received in-hospital services and medical treatment for hypo-/hyperthyroidism or had a medical certificate with an ICD9-CM code for TD (including thyroid cancers). Uncertain TD cases were confirmed with phone-call to patients.

Patients' demographic, epidemiological, clinical and laboratory (biochemical and viro-immunological) data were recorded in an electronic file. Information about coinfections, eventual comorbidities and medication history (including cART) were also collected. In particular, the following parameters were recorded at TD diagnosis and at the last available visit performed at our Clinic: HIV-RNA (cut-off of 37 copies/ml) and lymphocyte T CD4+ and CD8+ cell counts.

The outcomes of the research were TDs, considered in general and as specified diagnosis-related groups (hypothyroidism, hyperthyroidism and thyroid tumors). They were chosen arbitrarily a priori, considering scientific evidence and convenience to retrieve data.

Statistical analysis. Continuous variables were reported as mean with standard deviation $( \pm S D)$, categorical variables as frequency with percentages. We used chi-squared tests and Fisher exact tests for dichotomous variables comparison and t-test or Wilcoxon rank-sum test for group means comparison (for normal and non-normal distributions, respectively).

Odds ratios (ORs) were computed through multivariate logistic regression. Our model computed ORs for all outcomes, considering CD4+ nadir (as a dichotomous variable with cut-off: $200 \mathrm{cell} / \mathrm{mm}^{3}$ ), gender, age at HIV test (per 10-years older) and HCV coinfection. All the OR estimates were reported together with their $95 \%$ confidence intervals (95\% CIs) and the p value.

We performed the incidence rate analysis for TDs (considered all together) using periods of three years, from 2005 to 2016 . Analysis were stratified by gender. Subjects with less than 30 days of follow up were excluded from the incidence analysis but kept in all the others. We excluded prevalent cases. For this analysis we did not include incident cases during 2017 for getting homogeneous periods.

Moreover, two sensitivity analyses excluding patients with positive HCV antibodies and patients with an AIDS-defining diagnosis were conducted for both the incidence and the prevalence of TDs.

All statistical tests were two-sided at the level of significance $p<0.05$. Analyses were performed using Stata software version 14.0 (StataCorp, College Station, TX, USA).

Ethics. This study was conducted according to the Declaration of Helsinki and the principles of Good Clinical Practice (GCP). As this study had a retrospective design and was based on routinely collected data, patients' informed consent was not required according to the Italian law (Italian Guidelines for classification and conduction of observational studies, established by the Italian Drug Agency, "Agenzia Italiana del Farmaco - AIFA" on March 20,2008). Moreover, for this study we used the general authorization of the Italian Guarantor for the use of retrospective demographical and clinical data, which have been treated according to present laws. 


\begin{tabular}{|c|c|c|c|c|}
\hline & $\begin{array}{l}\text { NTD } \\
n=6220\end{array}$ & TD $n=123$ & $\begin{array}{l}\text { Total } \\
n=6343\end{array}$ & $\mathbf{p}$ \\
\hline \multirow{9}{*}{$\begin{array}{l}\text { Age at the end of follow up, mean years (SD) } \\
\text { Male gender, } n(\%) \\
\text { HCV Ab positive, } n(\%) \\
\text { HBsAg positive, } n(\%) \\
\text { AIDS, } n(\%) \\
\text { Last T CD4+ } \geq 200 / \mathrm{mm}^{3}, n(\%) \\
\text { Last viral load } \geq 50 \mathrm{cp} / \mathrm{ml}, \mathrm{n}(\%) \\
\text { Age at TD diagnosis, mean years (SD) } \\
\text { T CD } 4+\text { at TD diagnosis } \geq 200 / \mathrm{mm}^{3}, \mathrm{n}(\%)\end{array}$} & $47.30(10.98)$ & $47.15(11.56)$ & $47.3(11.9)$ & 0.84 \\
\hline & $4483(72.07)$ & $64(52.03)$ & $4547(71.68)$ & $<0.001$ \\
\hline & $2429(39.05)$ & $50(40.65)$ & $2479(39.08)$ & 0.81 \\
\hline & $528(8.49)$ & $14(11.38)$ & $542(8.54)$ & 0.33 \\
\hline & $4549(73.13)$ & $88(71.54)$ & $4637(73.10)$ & 0.70 \\
\hline & $5934(95.40)$ & $111(90.24)$ & 6045 (95.30) & 0.12 \\
\hline & $419(6.74)$ & $6(4.88)$ & $425(6.70)$ & $<0.001$ \\
\hline & NA & $46.6(11.5)$ & NA & \\
\hline & NA & 107 (86.99) & NA & \\
\hline
\end{tabular}

Table 1. Characteristics of the study population. TD: thyroid disease; NTD: no thyroid disease; SD: standard deviation; NA: not applicable.

\section{Results}

Characteristics of study cohort. From 2005 to 2017, 6343 HIV-infected patients have been followed at our Department with at least one visit; $71.68 \%$ was represented by males, mean age at the end of follow-up was $47.30 \pm 11.90$ years. HCV/HIV coinfection was present in $39.08 \%$ of the cohort. A total of 123 patients showed a symptomatic TD in our HIVeDB during the study period (1.94\% of the entire cohort including prevalent and incident cases). All detected subjects were confirmed by the HPADB and no additional cases were identified using this source.

Characteristics of patients with TD $(n=123)$. All patients were known to be HIV-infected when they developed thyroid dysfunction; mean age at TD diagnosis was 46.6 years (SD: 11.5). Characteristics of the study population at baseline and at the end of follow-up are summarized in Table 1 . In the TDs group, almost half of patients were females $(\mathrm{n}=59,48 \%), 67$ subjects $(54.5 \%)$ showed a CD4+ lymphocytes nadir $<200 / \mathrm{mm}^{3}$ and $71.54 \%(\mathrm{n}=88)$ experienced a previous AIDS-defining event. Mean time from HIV to TD diagnosis was 11.04 years (SD: 9.60); at TD diagnosis, mean T CD4+ cell count was 491 cell/uL (range 1-1556 cell/uL) and most patients had undetectable HIV-RNA $(\mathrm{n}=117,95.12 \%)$. The logistic regression analysis showed that TDs were negatively associated with male gender $(\mathrm{OR}=0.52,95 \%$ CI $0.35-0.77$; $\mathrm{p}<0.001)$ and positively associated with age at HIV test per 10 -year increase $(\mathrm{OR}=1.24,95 \%$ CI 1.06-1.45; $\mathrm{p}=0.005$ ). At the end of follow-up, all patients with TD had serum thyroid stimulating hormone (TSH), serum triiodothyronine (T3) and serum thyroxine (T4) levels within the reference ranges (data not shown). Overall, 99 patients $(80.49 \%)$ received levothyroxine, 17 (13.82\%) were cured with methimazole, $3(2.44 \%)$ took iodine and selenium supplements. Furthermore, 20 patients $(16.26 \%)$ had a history of surgical thyroidectomy. Figure 1 shows the distribution of TDs in the cohort considered. Hashimoto's thyroiditis and Graves' disease were the most frequent pathologies of thyroid gland (51.22\% and $13.82 \%$ of all TDs, respectively). Demographic and clinical characteristics of patients with TDs (classified in hypothyroidisms, hyperthyroidisms and TCs) are shown in Table 2. In this analysis we did not include 10/123 patients who suffered from an euthyroid goiter.

Patients with hypothyroidism $(\mathbf{n}=\mathbf{8 1})$. Hypothyroidism was the most prevalent TD in our cohort ( 81 out of $113,71.68 \%)$; the majority of patients $(n=63,77.78 \%)$ had a Hashimoto's thyroiditis. Mean age was $45.80 \pm 11.49$ years; slightly more than half was represented by males $(n=42,51.85 \%)$ and overall $74.07 \%$ of subjects presented at least one comorbidity. Concerning HIV status, 22 patients (27.16\%) experienced a previous AIDS-defining event, with a mean time from HIV acquisition to TD diagnosis of 11.23 years (SD: 10.20). At TD diagnosis all patients with hypothyroidism were on stable cART, the majority with a PI-containing regimen $(\mathrm{n}=69,85.19 \%)$. At the end of follow-up, all patients with hypothyroidism received a levothyroxine-based treatment. At the multivariate regression logistic analysis, hypothyroidism was negatively associated with male gender $(\mathrm{OR}=0.41,95 \% \mathrm{CI} 0.26-0.65 ; \mathrm{p}<0.001)$ while age at HIV test per 10 -year increase was borderline positively associated $(\mathrm{OR}=1.21,95 \% \mathrm{CI} 1.00-1.46 ; \mathrm{p}=0.05)$.

Patients with hyperthyroidism $(\mathbf{n}=\mathbf{2 1})$. The $18.5 \%(21 / 113)$ of patients with TD showed a symptomatic hyperthyroidism; the majority of them $(n=17,80.95 \%)$ suffered from Graves' disease. Males represented $52.38 \%$ of this group $(\mathrm{n}=11)$; mean age was 42.52 years (SD: 12.58). $76.19 \%$ of patients presented at least one comorbidity. Mean time from HIV to TD diagnosis was 12.06 years (SD: 8.14), mean T CD4+ nadir was 105.74 cell/ $\mathrm{mm}^{3}$ (SD: 169.30). At TD diagnosis the totality of patients with hyperthyroidism received cART, $57.14 \%(\mathrm{n}=$ 512) with a PI-based regimen. At the end of study, all patients were treated for their thyroid dysfunction (17 with methimazole, 4 with levothyroxine), while 5 underwent thyroidectomy. At the multivariate regression logistic analysis, only T CD4+ cell nadir $<200 \mathrm{cell} / \mathrm{mm}^{3}$ was independently associated with hyperthyroidism symptomatic disorders $(\mathrm{OR}=3.08,95 \%$ CI $1.06-1.45 ; \mathrm{p}=0.005)$.

Patients with primitive thyroid cancer $(\mathbf{n}=\mathbf{1 1})$. Eleven subjects were diagnosed with TC during the study period. Papillary thyroid cancer was the most frequent TC $(n=7,63.63 \%)$, followed by medullary $(n=2$, $18.18 \%)$ and follicular thyroid cancer $(n=1,9.1 \%)$. The histotype was not recorded for one patient. In this group, 
THYROID DISEASES $(n=123)$

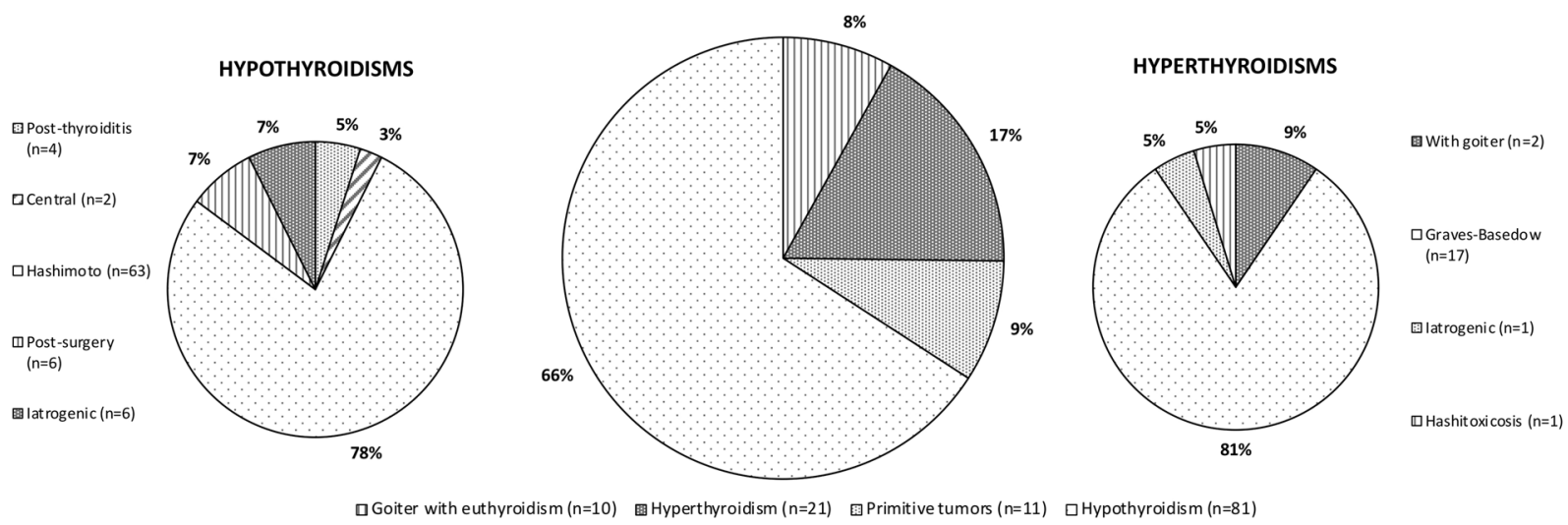

Figure 1. Distribution of symptomatic thyroid diseases (TDs) in the cohort considered.

\begin{tabular}{|c|c|c|c|}
\hline & $\begin{array}{l}\text { Hypothyroidism } \\
\mathrm{n}=81\end{array}$ & $\begin{array}{l}\text { Hyperthyroidism } \\
\mathrm{n}=21\end{array}$ & $\begin{array}{l}\text { Primitive Thyroid } \\
\text { Cancers } n=11\end{array}$ \\
\hline Male gender, $\mathrm{n}(\%)$ & $42(51.85)$ & $11(52.38)$ & $6(54.54)$ \\
\hline Age at TD diagnosis, mean years (SD) & $45.80(11.49)$ & $42.52(12.58)$ & $48.64(6.30)$ \\
\hline HCV Ab positive, $n(\%)$ & $30(37.04)$ & $9(42.86)$ & $4(36.36)$ \\
\hline HBsAg positive, $\mathrm{n}(\%)$ & $4(4.94)$ & $3(14.29)$ & $2(18.18)$ \\
\hline Delay from HIV diagnosis to TD onset, years (SD) & $11.23(10.20)$ & $12.06(8.14)$ & $8.69(9.88)$ \\
\hline Time from cART start to TD onset, years (SD) & $6.03(9.07)$ & $7.96(6.12)$ & $5.67(5.85)$ \\
\hline $\mathrm{CD} 4+\mathrm{T}$ cell nadir $/ \mathrm{mm}^{3}, \mathrm{n}(\mathrm{SD})$ & $199.54(162.34)$ & $105.74(169.30)$ & $174.36(106.34)$ \\
\hline $\mathrm{CD} 4+/ \mathrm{CD} 8+(\mathrm{SD})$ & $0.33(0.40)$ & $0.29(0.31)$ & $0.17(0.14)$ \\
\hline CD4+ T cell increase from nadir to TD diagnosis, $\mathrm{n}(\mathrm{SD})$ & $288.89(255.77)$ & $437.54(306.13)$ & $316.20(304.01)$ \\
\hline AIDS, n (\%) & $22(27.16)$ & $11(52.38)$ & $2(18.18)$ \\
\hline Comorbities $\geq 1, \mathrm{n}(\%)$ & $60(74.07)$ & $16(76.19)$ & $10(90.91)$ \\
\hline Hypertension & $27(33.33)$ & $9(42.86)$ & $5(45.45)$ \\
\hline Osteopenia/osteoporosis & $18(22.22)$ & $3(14.29)$ & $3(27.27)$ \\
\hline Diabetes mellitus & $15(18.52)$ & $4(19.05)$ & $2(18.18)$ \\
\hline \multicolumn{4}{|l|}{ cART at TD diagnosis } \\
\hline INI + NRTI, n (\%) & $2(2.47)$ & $6(28.57)$ & $2(18.18)$ \\
\hline PI + NRTI, n (\%) & $69(85.19)$ & $12(57.14)$ & $1(9.09)$ \\
\hline NNRTI + NRTI, n (\%) & $6(7.41)$ & $2(9.52)$ & $3(27.27)$ \\
\hline Other, n (\%) & $4(4.94)$ & $1(4.76)$ & $5(45.45)$ \\
\hline
\end{tabular}

Table 2. Demographic, clinical and immunological characteristics of patients with symptomatic thyroid diseases $(n=113)$, classified in three groups as follows: hypothyroidisms, hyperthyroidisms and primitive thyroid cancers. SD: standard deviation; TD: thyroid disease; cART: combined antiretroviral therapy; INI: integrase inhibitor; NRTI: nucleoside reverse transcriptase inhibitor; PI: protease inhibitor; NNRTI: nonnucleoside reverse transcriptase inhibitor.

mean age was 48.64 years (SD: 6.30) and patients were equally divided by gender ( $45.5 \%$ females). Almost all subjects $(\mathrm{n}=10,90.91 \%)$ presented at least one comorbidity (cardiovascular, diabetes or osteopenia), while $36.36 \%$ $(\mathrm{n}=4)$ were coinfected with HCV. TC was meanly diagnosed after 8.69 years from HIV diagnosis (SD: 9.88$)$ and $100 \%$ of patients was on stable cART at that time (63.64\% showing an undetectable viremia, with a mean T CD4+ cell count of $527 \mathrm{cell} / \mathrm{mm}^{3}$ ). All subjects underwent thyroidectomy and all received a substitution therapy with sodic levothyroxine. One of the patients with papillary thyroid cancer had a metastatic disease involving lungs and bones; he finally died, after six years of chemotherapies and radiotherapies. TC diagnosis was made at 47 years old; patient remained in good viro-immunological conditions until the exitus (at the last exams available: HIVRNA not detectable, T CD $4+510 \mathrm{cell} / \mathrm{mm}^{3}$ ). To date, the rest of patients diagnosed with TC are in good conditions. At the multivariate logistic regression analysis TC diagnosis was independently associated only with age at HIV test per 10-year old increase $(\mathrm{OR}=1.68,95 \% \mathrm{CI} 1.05-2.66 ; \mathrm{p}=0.03)$.

Incidence of thyroid diseases from 2005 to 2016 in our cohort. For the incidence analysis we limited the period from 2005 to 2016 . We included 5337 patients with at least 2 available visits during this period, excluding prevalent cases (42251.71 person-years of follow-up). We observed 96 TDs incident cases with an overall 


\begin{tabular}{|l|l|l|l|l|l|l|}
\hline \multirow{2}{*}{ Years } & Gender & $\begin{array}{l}\text { Total subjects } \\
(\mathbf{n}, \mathbf{\%})\end{array}$ & $\begin{array}{l}\text { Mean age } \\
( \pm \mathbf{S D})\end{array}$ & $\begin{array}{l}\text { TD diagnosis } \\
(\mathbf{n})\end{array}$ & $\begin{array}{l}\text { Person-years } \\
\text { of follow-up }\end{array}$ & $\begin{array}{l}\text { Incidence rate } \\
(\mathbf{9 5 \%} \text { CI })\end{array}$ \\
\hline \multirow{2}{*}{$2005-2016$} & $\mathrm{~F}$ & $1502(28.14)$ & $41.15(10.37)$ & 46 & 11865.62 & $3.88(2.91-5.18)$ \\
\cline { 2 - 7 } & $\mathrm{M}$ & $3835(71.86)$ & $44.49(9.69)$ & 50 & 30386.1 & $1.64(1.24-2.17)$ \\
\hline \multirow{2}{*}{$2005-2007$} & $\mathrm{~F}$ & $1086(27.97)$ & $38.88(9.03)$ & 6 & 2832.48 & $2.12(0.95-4.72)$ \\
\cline { 2 - 7 } & $\mathrm{M}$ & $2798(72.04)$ & $41.89(8.43)$ & 9 & 7228.32 & $1.25(0.65-2.39)$ \\
\hline \multirow{2}{*}{$2008-2010$} & $\mathrm{~F}$ & $1128(28.23)$ & $41.12(9.63)$ & 8 & 2986.03 & $2.68(1.34-5.36)$ \\
\cline { 2 - 7 } & $\mathrm{M}$ & $2868(71.77)$ & $44.30(8.81)$ & 10 & 7506.28 & $1.33(0.72-2.48)$ \\
\hline \multirow{2}{*}{$2011-2013$} & $\mathrm{~F}$ & $1271(28.40)$ & $42.63(10.52)$ & 17 & 3045.55 & $5.58(3.47-8.98)$ \\
\cline { 2 - 7 } & $\mathrm{M}$ & $3204(71.60)$ & $45.63(9.75)$ & 17 & 7768.06 & $2.19(1.36-3.52)$ \\
\hline \multirow{2}{*}{$2014-2016$} & $\mathrm{~F}$ & $1126(27.85)$ & $45.80(10.49)$ & 15 & 2993.36 & $5.01(3.02-8.31)$ \\
\cline { 2 - 7 } & $\mathrm{M}$ & $2917(72.15)$ & $48.56(9.78)$ & 14 & 7862.49 & $1.78(1.05-3.01)$ \\
\hline
\end{tabular}

Table 3. Thyroid diseases incidence rates for the entire follow-up considered (2005-2016) and for each 3-year period.

crude incidence rate of 2.2 per 1000 persons (95\% CI 1.8-2.7); the crude incidence rate was 1.6 per 1000 persons (95\% CI 1.2-2.2) for males and 3.8 per 1000 persons (95\% CI 2.9-5.1) for females. Demographics characteristics and TDs incidence rates for the entire follow-up considered (2005-2016) and for each 3-year period are shown in Table 3.

Temporal trend was also analyzed by Poisson regression as incident relative risk (IRR). For each 3-year period TDs incidence increased of 25\%, with an IRR of 1.25 (95\% CI 1.04-1.50; $p=0.02)$, mainly attributed to an incidence increase in females $(\mathrm{IRR}=1.16,95 \% \mathrm{CI} 0.90-1.49 ; \mathrm{p}=0.24$ for males and $\mathrm{IRR}=1.36,95 \% \mathrm{CI} 1.03-1.78$; $\mathrm{p}=0.03$ for females).

As expected, at the sensitivity analyses excluding subjects with positive HCV antibodies and with an AIDS-defining diagnosis we found only few differences for both the incidence and the prevalence of TDs due to the small number of total patients with symptomatic thyroid disorders considered (data not shown).

\section{Discussion}

Here we report that TDs, including cancers, are a rare event in PLWH, with double incidence for females compared to males. Thus, in our cohort, only $1.94 \%$ of HIV-infected patients were diagnosed with thyroid disturbances during the follow-up period: $1.28 \%$ showed symptomatic hypothyroidism, $0.33 \%$ symptomatic hyperthyroidism, while thyroid cancer was documented in $0.17 \%$ of the considered population.

Other studies reported a significative higher prevalence of TDs in PLWH, ranging from $16 \%$ to $33.1 \%{ }^{11,26-29}$, since they included also subclinical TDs in their analysis. If we compare the sole prevalence of overt hypo and hyperthyroidism in HIV-positive individuals, our data are similar to those found by other Authors, although their studies were performed in much smaller cohorts ${ }^{17,30-32}$. Moreover, in our cohort all patients were on stable cART at TD diagnosis, confirming that well treated PLWH are not at an increased risk of thyroid dysfunction, as previous described ${ }^{14-17}$.

However, it is not easy to make comparisons among distinct epidemiologic studies, due to the diversity of populations and end-points analyzed. Differences involve disease definition and severity (e.g. overt vs subclinical dysfunction), selection criteria, different reference ranges and laboratory techniques used to measure serum thyroid hormone levels ${ }^{33}$, besides the influence of age, sex and environmental factors ${ }^{34}$. For these reasons and considering the high prevalence of subclinical thyroid dysfunctions in HIV-infected subjects, we analyzed only symptomatic diseases.

The two major autoimmune TDs, Hashimoto's thyroiditis and Graves' disease, were the most frequent pathologies of thyroid gland in our cohort. Although small researches reported a prevalence of Hashimoto thyroiditis up to $2.6 \%$ in HIV-positive patients ${ }^{35}$, a large-population based study ${ }^{36}$ evaluated the presence of autoimmune diseases among $5186 \mathrm{HIV}$-infected patients, finding only 1 case of Hashimoto's thyroiditis and 2 cases of Graves' disease. Thus, these TDs seem to be more frequent in our cohort, showing a prevalence comparable to international general population values ${ }^{34}$.

Autoimmune TDs are much common among women than in men, with a female to male ratio ranging from $5: 1$ to $10: 1$ in general population ${ }^{34}$. The biological explanation for this gender difference is not entirely clarified, but the X chromosome inactivation could significantly contribute to the high incidence of autoimmune TDs in females ${ }^{37}$; moreover, it seems that maternal immune responses against fetal antigens can trigger autoimmune processes $^{38}$. In our cohort of PLWH, male gender was confirmed as a protective factor for TDs onset, especially for hypothyroidism.

TDs development is also influenced by ageing in general population ${ }^{39}$. In our study, age at HIV test emerged as a variable associated with both hypothyroidism and TCs. Notably, we also focused on temporal trends, finding an increase in TDs incidence for each 3-year period from 2005 to 2016, attributable to the ageing of our cohort. Thus, increasing age and non-AIDS related comorbidities (which were highly represented in our cohort, with a peak of $90.91 \%$ of prevalence for TCs), together with chronic inflammation, immunosenescence and polypharmacy may play a role in TDs occurrence and progression.

However, immune restoration is also probably implicated in TDs development. As a matter of fact, we found that a T CD 4 + cell nadir $<200 \mathrm{cell} / \mathrm{mm}^{3}$ was independently associated with symptomatic hyperthyroidism. In this group we reported the most remarkable CD4+ T cell increase from nadir to TD diagnosis (mean CD4+ 
increase: $437.54 \pm 306.13$ ), supporting the previously described hypothesis of Graves' disease as a manifestation of delayed immune reconstitution inflammatory syndrome (IRIS) ${ }^{21,22}$ and suggesting that some CD4+ T lymphocytes subsets may affect its occurrence ${ }^{40}$.

Finally, we did not find any correlation between HCV coinfection and thyroid abnormalities, although some Authors highlighted the role of both HBV and HCV in increasing the probability of thyroid dysfunction, especially hypothyroidism ${ }^{20,29}$.

In the post-HAART period, PLWH reported an increasing risk of non-AIDS-defining cancers that it is higher compared to uninfected controls ${ }^{41-43}$. To our knowledge, our study is the first one to evaluate TCs prevalence and associated risk factors in a population of HIV-infected patients. According to the latest Italian general population tumor report ${ }^{44}$, the risk to develop a TC is much higher in females compared to males; furthermore, although TC incidence is increasing, the overall mortality remains extremely low. In our study, TC seems to be a very rare condition, with a lower prevalence than the one reported for general population ( $0.17 \mathrm{vs} 0.33 \%$, respectively). In this population of PLWH, patients diagnosed with TC were equally divided by gender(although females represent less than $30 \%$ of the total cohort) and age was the only risk factor associated with thyroid tumor development. Italian data ${ }^{44}$ show that, from 2010 to $2014,82 \%$ of TCs was represented by papillary thyroid cancer, followed by follicular (7\%), medullary (4\%) and anaplastic (1\%) histotypes. As expected, in our cohort of PLWH, the most frequent TC was represented by papillary thyroid cancer. Interestingly, medullary thyroid cancer was diagnosed in almost $20 \%$ of patients considered in our study, with a percentage significantly higher compared to the Italian general population. Although medullary thyroid cancer development was associated with mutations of RET tyrosine kinase, no information is available about a possible interaction between HIV and thyroid tissue, as described for $\mathrm{EBV}^{45}$. However, due to our limited sample size, more researches are needed in order to confirm our results and to evaluate the real distribution of TCs in a population of HIV-infected individuals. A recent study ${ }^{46}$, performed using data from $>6$ million US patients from the National Cancer Data Base (NCDB), showed that PLWH were more likely to be diagnosed with advanced-stage of TCs and to experience a higher mortality after TC diagnosis compared to general population. At TC diagnosis, all individuals considered in our cohort were on stable cART, the majority with a satisfying viro-immunological profile; moreover, at the end of follow-up, TC was controlled in all patients except one, who died.

Our study should be interpreted within its limitations, firstly the retrospective monocentric design, which does not permit a more comprehensive description of HIV and immune system dynamicity. Secondly, although every TD diagnosis was accurately verified, there could be some bias in estimating the prevalence of thyroid disturbances in the population considered. Finally, all participants are from the same geographical area and it is reasonable to assume a similar iodine intake; however, our results may not apply to regions with different iodine consumption.

The strengths of this study include the large population size, the enrolment of an unselected group of HIV-infected patients and an accurate retrieval of data on TDs thanks to the use of two different sources, the clinic database and the HPADB of Brescia Province.

\section{Conclusions}

To conclude, our study is the first to analyze the prevalence of exclusively symptomatic TDs (with accurate retrieval of diagnosis) in a large cohort of HIV-infected patients, confirming age and gender as crucial elements in the onset of thyroid abnormalities and highlighting the role of T CD4+ cell nadir as additional factor. Despite the high prevalence of thyroid dysfunction described in PLWH, symptomatic TDs remain a rare event. However, in the next decades, we will probably face a progressive increase of this comorbidity, considering that TDs (especially hypothyroidism) are dramatically more prevalent after the age of $40^{47}$ and that PLWH are actually ageing. Periodic screening with measurement of TSH levels could be implemented, in order to provide a rapid treatment and to minimize thyroid-related complications.

Moreover, we found an interesting much more elevated rate of medullary thyroid cancer in PLWH compared to the general population; however, the distribution of different histotypes and their extensive characterizations are yet to be fully described in this category of patients.

Received: 15 July 2019; Accepted: 5 December 2019;

Published online: 19 December 2019

\section{References}

1. Grinspoon, S. K., Donovan, D. S. Jr. \& Bilezikian, J. P. Aetiology and pathogenesis of hormonal and metabolic disorders in HIV infection. Baillieres. Clin Endocrinol Metab. 8, 735-755 (1994).

2. Hofbauer, L. C. \& Heufelder, A. E. Endocrine implications of human immunodeficiency virus infection. Medicine (Baltimore). 75, 262-278 (1996)

3. Mirza, F. S., Luthra, P. \& Chirch, L. Endocrinological aspects of HIV infection. J Endocrinol Invest. 41, 881-899 (2018).

4. Fried, J. C. et al. Serum triiodothyronine values. Prognostic indicators of acute mortality due to Pneumocystis carinii pneumonia associated with the acquired immunodeficiency syndrome. Arch Intern Med. 150, 406-409 (1990).

5. Grunfeld, C. et al. Indices of thyroid function and weight loss in human immunodeficiency virus infection and the acquired immunodeficiency syndrome. Metabolism. 42, 1270-1276 (1993).

6. Sellmeyer, D. E. \& Grunfeld, C. Endocrine and metabolic disturbances in human immunodeficiency virus infection and the acquired immune deficiency syndrome. Endocr Rev. 17, 518-532 (1996).

7. Madeddu, G. et al. Thyroid function in human immunodeficiency virus patients treated with highly active antiretroviral therapy (HAART): a longitudinal study. Clin Endocrinol (Oxf). 64, 375-383 (2006).

8. Silva, G. A. et al. Association between antiretrovirals and thyroid diseases: a cross-sectional study. Arch Endocrinol Metab. 59, 116-122 (2015).

9. Hoffmann, C. J. \& Brown, T. T. Thyroid function abnormalities in HIV-infected patients. Clin Infect Dis. 45, 488-494 (2007). 
10. Collazos, J., Ibarra, S. \& Mayo, J. Thyroid hormones in HIV-infected patients in the highly active antiretroviral therapy era: evidence of an interrelation between the thyroid axis and the immune system. AIDS. 17, 763-765 (2003).

11. Beltran, S. et al. Increased prevalence of hypothyroidism among human immunodeficiency virus-infected patients: a need for screening. Clin Infect Dis. 37, 579-583 (2003).

12. Grappin, M. et al. Increased prevalence of subclinical hypothyroidism in HIV patients treated with highly active antiretroviral therapy. AIDS. 14, 1070-1072 (2000).

13. Bongiovanni, M. et al. Subclinical hypothyroidism in HIV-infected subjects. J Antimicrob Chemother. 58, 1086-1089 (2006).

14. Carvalho, L. G. et al. Evaluation of thyroid function and autoimmunity in HIV-infected women. Arq Bras Endocrinol Metabol. 58, 86 (2014).

15. Wiener, M., Lo, Y. \& Klein, R. Abnormal thyroid function in older men with or at risk for HIV infection. HIV Med. 9, 544-549 (2008).

16. Madge, S. et al. No association between HIV disease and its treatment and thyroid function. HIV Med. 8, 22-27 (2007).

17. Harsløf, M. et al. No evidence of increased risk of thyroid dysfunction in well treated people living with HIV. AIDS. 32, 2195-2199 (2018).

18. Calza, L., Manfredi, R. \& Chiodo, F. Subclinical hypothyroidism in HIV-infected patients receiving highly active antiretroviral therapy. J. Acquir Immune Defic Syndr. 31, 361-363 (2002).

19. Beltran, S., Lescure, F. X., El Esper, I., Schmit, J. L. \& Desailloud, R. Subclinical hypothyroidism in HIV-infected patients is not an autoimmune disease. Horm Res. 66, 21-26 (2006).

20. Welch, K. et al. Autopsy findings in the acquired immune deficiency syndrome. JAMA. 252, 1152-1159 (1984).

21. Chen, F. et al. Characteristics of autoimmune thyroid disease occurring as a late complication of immune reconstitution in patients with advanced human immunodeficiency virus (HIV) disease. Medicine (Baltimore). 84, 98-106 (2005).

22. Jubault, V. et al. Sequential occurrence of thyroid autoantibodies and Graves' disease after immune restoration in severely immunocompromised human immunodeficiency virus-1-infected patients. J Clin Endocrinol Metab. 85, 4254-4257 (2000).

23. Di Domenicantonio, A. et al. A review on thyroid autoimmune disorders and HCV chronic infection. Clin Ter. 165, e376-381 (2014).

24. Fallahi, P., Ferrari, S. M., Vita, R., Benvenga, S. \& Antonelli, A. The role of human parvovirus B19 and hepatitis C virus in the development of thyroid disorders. Rev Endocr Metab Disord. 17, 529-535 (2016).

25. Muratori, L. et al. Susceptibility to thyroid disorders in hepatitis C. Clin Gastroenterol Hepatol. 3, 595-603 (2005).

26. Raffi, F. et al. Endocrine function in $98 \mathrm{HIV}$-infected patients: a prospective study. AIDS. 5, 729-733 (1991).

27. Báez, M. S., Zapata Silva, A., López Benavides, M. I. \& Wilson, G. Thyroid dysfunction among HIV infected patients. Rev Med Chil. 144, 333-340 (2016).

28. Ketsamathi, C., Jongjaroenprasert, W., Chailurkit, L. O., Udomsubpayakul, U. \& Kiertiburanakul, S. Prevalence of thyroid dysfunction in Thai HIV-infected patients. Curr HIV Res. 4, 463-467 (2006).

29. Ji, S. et al. Prevalence and Influencing Factors of Thyroid Dysfunction in HIV-Infected Patients. Biomed Res Int. 2016, 3874257 (2016).

30. Noureldeen, A. F., Qusti, S. Y. \& Khoja, G. M. Thyroid function in newly diagnosed HIV-infected patients. Toxicol Ind Health. 30 , 919-925 (2014).

31. Sharma, N. et al. Prevalence and Predictors of Thyroid Dysfunction in Patients with HIV Infection and Acquired Immunodeficiency Syndrome: An Indian Perspective. J Thyroid Res. 2015, 517173 (2015).

32. Sebastian, S. A., Sumithra, S., Kurian, J., Mathew, V. \& Idiculla, J. M. Thyroid dysfunction in patients on antiretroviral therapy: A perspective from southern India. Natl Med J India. 31, 136-139 (2018).

33. Vanderpump, M. P. et al. The incidence of thyroid disorders in the community: a twenty-year follow-up of the Whickham Survey. Clin Endocrinol (Oxf). 43, 55-68 (1995).

34. Garmendia Madariaga, A., Santos Palacios, S., Guillén-Grima, F. \& Galofré, J. C. The incidence and prevalence of thyroid dysfunction in Europe: a meta-analysis. J Clin Endocrinol Metab. 99, 923-931 (2014).

35. Parsa, A. A. \& Bhangoo, A. HIV and thyroid dysfunction. Rev Endocr Metab Disord. 14, 127-131 (2013).

36. Virot, E. et al. Autoimmune diseases and HIV infection: A cross-sectional study. Medicine (Baltimore). 96, e5769 (2017).

37. Simmonds, M. J. et al. Skewed $\mathrm{x}$ chromosome inactivation and female preponderance in autoimmune thyroid disease: an association study and meta-analysis. J Clin Endocrinol Metab. 99, e127-131 (2014).

38. Lepez, T. et al. Fetal microchimeric cells in blood of women with an autoimmune thyroid disease. PLoS One. 6, e29646 (2011)

39. Hollowell, J. G. et al. Serum TSH, T4, and thyroid antibodies in the United States population (1988 to 1994): National Health and Nutrition Examination Survey (NHANES III). J Clin Endocrinol Metab. 87, 489-499 (2002).

40. Quiros-Roldan, E. et al. Peripheral loss of regulatory T cells and polyautoimmunity in an HIV-infected patient. Int J STD AIDS. 29, $1345-1347$ (2018).

41. Raffetti, E. et al. Cancer incidence and mortality for all causes in HIV-infected patients over a quarter century: a multicentre cohort study. BMC Public Health. 15, 235 (2015).

42. Nagata, N. et al. Increased risk of non-AIDS-defining cancers in Asian HIV-infected patients: a long-term cohort study. BMC Cancer. 18, 1066 (2018).

43. Rubinstein, P. G., Aboulafia, D. M. \& Zloza, A. Malignancies in HIV/AIDS: from epidemiology to therapeutic challenges. AIDS. 28, 453-465 (2014)

44. Gori, S. et al. I numeri del cancro in Italia, available at, https://www.registri-tumori.it/cms/sites/default/files/pubblicazioni/2018_ NumeriCancro-operatori.pdf (2018).

45. Moghoofei, M. et al. Epstein-Barr virus and thyroid cancer: The role of viral expressed proteins. J Cell Physiol. 234, 3790-3799 (2019).

46. Coghill, A. E. et al. Advanced stage at diagnosis and elevated mortality among US patients with cancer infected with HIV in the National Cancer Data Base. Cancer. 125, 2868-2876 (2019).

47. Laurberg, P., Bülow Pedersen, I., Pedersen, K. M. \& Vestergaard, H. Low incidence rate of overt hypothyroidism compared with hyperthyroidism in an area with moderately low iodine intake. Thyroid. 9, 33-38 (1999).

\section{Author contributions}

E.Q.R. and M.P. conceived the study. M.P., T.G., N.G. and A.C. made substantial contributions to the acquisition of patients' clinical data. T.G. and S.M. performed statistical analysis. M.P. and E.Q.R. wrote the first version of the manuscript. A.C. and N.G. prepared Fig. 1 and Tables 1, 2 and 3. F.C. contributed to the design and implementation of the research, to the analysis of results and to the writing of the manuscript. All authors revised critically for important intellectual content. All authors read and approved the final manuscript.

\section{Competing interests}

The authors declare no competing interests. This work was entirely supported by internal founds. 
Additional information

Correspondence and requests for materials should be addressed to M.P.

Reprints and permissions information is available at www.nature.com/reprints.

Publisher's note Springer Nature remains neutral with regard to jurisdictional claims in published maps and institutional affiliations.

(c) (i) Open Access This article is licensed under a Creative Commons Attribution 4.0 International License, which permits use, sharing, adaptation, distribution and reproduction in any medium or format, as long as you give appropriate credit to the original author(s) and the source, provide a link to the Creative Commons license, and indicate if changes were made. The images or other third party material in this article are included in the article's Creative Commons license, unless indicated otherwise in a credit line to the material. If material is not included in the article's Creative Commons license and your intended use is not permitted by statutory regulation or exceeds the permitted use, you will need to obtain permission directly from the copyright holder. To view a copy of this license, visit http://creativecommons.org/licenses/by/4.0/.

(C) The Author(s) 2019 